\title{
Front Matter: Volume 7870
}

, "Front Matter: Volume 7870," Proc. SPIE 7870, Image Processing: Algorithms and Systems IX, 787001 (25 February 2011); doi: 10.1117/12.890408

EPIE Event: IS\&T/SPIE Electronic Imaging, 2011, San Francisco Airport, California, SPIE. United States 
PROCEEDINGS

IS\&T / SPIE

Electronic

Imaging

SCIENCE AND TECHNOLOGY

\section{Image Processing: Algorithms and Systems IX}

Jaakko T. Astola

Karen O. Egiazarian

Editors

24-25 January 2011

San Francisco, California, United States

Sponsored and Published by

IS\&T-The Society for Imaging Science and Technology

SPIE 
The papers included in this volume were part of the technical conference cited on the cover and title page. Papers were selected and subject to review by the editors and conference program committee. Some conference presentations may not be available for publication. The papers published in these proceedings reflect the work and thoughts of the authors and are published herein as submitted. The publisher is not responsible for the validity of the information or for any outcomes resulting from reliance thereon.

Please use the following format to cite material from this book:

Author(s), "Title of Paper," in Image Processing: Algorithms and Systems IX, edited by Jaakko T. Astola, Karen O. Egiazarian, Proceedings of SPIE-IS\&T Electronic Imaging, SPIE Vol. 7870 (SPIE, Bellingham, WA, 2011) Article CID Number.

ISSN 0277-786X

ISBN 9780819484079

Published by

SPIE

P.O. Box 10, Bellingham, Washington 98227-0010 USA

Telephone +1 3606763290 (Pacific Time) · Fax +1 3606471445

SPIE.org

and

IS\&T-The Society for Imaging Science and Technology

7003 Kilworth Lane, Springfield, Virginia, 22151 USA

Telephone +1 7036429090 (Eastern Time) · Fax +1 7036429094

imaging.org

Copyright () 2011, Society of Photo-Optical Instrumentation Engineers and The Society for Imaging Science and Technology

Copying of material in this book for internal or personal use, or for the internal or personal use of specific clients, beyond the fair use provisions granted by the U.S. Copyright Law is authorized by SPIE subject to payment of copying fees. The Transactional Reporting Service base fee for this volume is $\$ 18.00$ per article (or portion thereof), which should be paid directly to the Copyright Clearance Center (CCC), 222 Rosewood Drive, Danvers, MA 01923. Payment may also be made electronically through CCC Online at copyright.com. Other copying for republication, resale, advertising or promotion, or any form of systematic or multiple reproduction of any material in this book is prohibited except with permission in writing from the publisher. The CCC fee code is 0277-786X/11/ \$18.00.

Printed in the United States of America.

Publication of record for individual papers is online in the SPIE Digital Library.

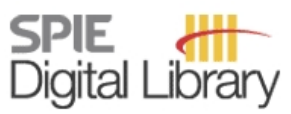

SPIEDigitalLibrary.org

Paper Numbering: Proceedings of SPIE follow an e-First publication model, with papers published first online and then in print and on CD-ROM. Papers are published as they are submitted and meet publication criteria. A unique, consistent, permanent citation identifier (CID) number is assigned to each article at the time of the first publication. Utilization of CIDs allows articles to be fully citable as soon they are published online, and connects the same identifier to all online, print, and electronic versions of the publication. SPIE uses a six-digit CID article numbering system in which:

- The first four digits correspond to the SPIE volume number.

- The last two digits indicate publication order within the volume using a Base 36 numbering system employing both numerals and letters. These two-number sets start with 00, 01, 02, 03, 04 , $05,06,07,08,09,0 A, 0 B \ldots 0 Z$, followed by 10-1Z, 20-2Z, etc.

The CID number appears on each page of the manuscript. The complete citation is used on the first page, and an abbreviated version on subsequent pages. Numbers in the index correspond to the last two digits of the six-digit CID number. 


\section{Contents}

vii Conference Committee

\section{SESSION 1 IMAGE FILTERING AND ENHANCEMENT}

787003 Video denoising using separable 4D nonlocal spatiotemporal transforms [7870-02] M. Maggioni, Tampere Univ. of Technology (Finland); G. Boracchi, Politecnico di Milano (Italy); A. Foi, K. Egiazarian, Tampere Univ. of Technology (Finland)

787004 Intelligent edge enhancement using multilayer neural network based on multi-valued neurons [7870-03]

I. Aizenberg, S. Alexander, J. Jackson, T. Neal, J. Wilson, K. Kendrick, Texas A\&M Univ. (United States)

787005 Error minimizing algorithms for nearest neighbor classifiers [7870-04]

R. B. Porter, D. Hush, Los Alamos National Lab. (United States); G. B. Zimmer, Texas A\&M Univ. (United States)

787006 Signal filtering of daily cloud types' trends as derived from satellite images [7870-05]

J. R. Dim, H. Murakami, Japan Aerospace Exploration Agency (Japan)

\section{SESSION 2 IMAGE ANALYSIS}

787007 Analysing wear in carpets by detecting varying local binary patterns [7870-06]

S. A. Orjuela, E. Vansteenkiste, F. Rooms, S. De Meulemeester, R. de Keyser, W. Philips, Ghent Univ. (Belgium)

787008 Line and streak detection on polished and textured surfaces using line integrals [7870-07] M. S. Erkilinc, M. Jaber, E. Saber, R. Pearson, Rochester Institute of Technology (United States)

787009 Spatio-temporal analysis and forward modeling of solar polar plumes in white light [7870-09] A. Llebaria, O. Morillot, LAM-OAMP, CNRS, Univ. de Provence (France); Y. Boursier, Ctr. de Physique des Particules de Marseille (France); P. Lamy, LAM-OAMP, CNRS, Univ. de Provence (France)

7870 OA Detecting photographic and computer generated composites [7870-08]

V. Conotter, L. Cordin, Univ. of Trento (Italy)

$7870 \mathrm{OB} \quad$ Imaging using synchrotron radiation for forensic science [7870-10]

F. Cervelli, S. Carrato, Univ. degli Studi di Trieste (Italy); A. Mattei, Reparto Investigazioni Scientifiche dei Carabinieri (Italy); M. Jerian, Amped (Italy); L. Benevoli, L. Mancini, F. Zanini, L. Vaccari, A. Perucchi, G. Aquilanti, Sincrotrone Trieste (Italy) 
7870 OC PSO-based methods for medical image registration and change assessment of pigmented skin [7870-11]

S. Kacenjar, Lockheed Martin Corp. (United States); M. Zook, Fox Chase Cancer Ctr. (United States); M. Balint, Lockheed Martin Corp. (United States)

7870 OD Image-based segmentation for characterization and quantitative analysis of the spinal cord injuries by using diffusion patterns [7870-12]

M. Hannula, A. Olubamiji, I. Kunttu, Tampere Univ. of Technology (Finland); P. Dastidar, S. Soimakallio, J. Öhman, Tampere Univ. Hospital (Finland); J. Hyttinen, Tampere Univ. of Technology (Finland)

7870 OE Descreening using segmentation-based adaptive filtering [7870-13]

M. N. Ahmed, A. H. Eid, Lexmark International, Inc. (United States)

\section{SESSION $4 \quad$ IMAGE TRANSFORMS AND APPLICATIONS}

$78700 \mathrm{~S}$ Secure annotation for medical images based on reversible watermarking in the Integer Fibonacci-Haar transform domain [7870-16]

F. Battisti, M. Carli, A. Neri, Univ. degli Studi di Roma Tre (Italy)

$7870 \mathrm{OH} \quad$ Multi-seam carving via seamlets [7870-17]

D. D. Conger, Michigan State Univ. (United States); M. Kumar, Eastman Kodak Co. (United States); H. Radha, Michigan State Univ. (United States)

7870 이 A new DCT-based algorithm for numerical reconstruction of electronically recorded holograms [7870-18]

L. Bilevich, L. Yaroslavsky, Tel Aviv Univ. (Israel)

\section{SESSION $5 \quad$ IMAGE PROCESSING SYSTEMS}

7870 0J User discrimination in automotive systems [7870-20]

A. Makrushin, J. Dittmann, Otto-von-Guericke-Univ. Magdeburg (Germany); C. Vielhaver, Otto-von-Guericke-Univ. Magdeburg (Germany) and Univ. of Applied Sciences

Brandenburg (Germany); M. Leich, Otto-von-Guericke-Univ. Magdeburg (Germany)

\section{SESSION $6 \quad$ IMAGE INTERPOLATION AND RECONSTRUCTION}

$7870 \mathrm{OL} \quad$ Wiener crosses borders: interpolation based on second order models [7870-22]

A. Guevara, R. Mester, Goethe Univ. (Germany)

7870 OM Image interpolation based on a multi-resolution directional map [7870-23]

E. Van Reeth, STMicroelectronics (France) and GIPSA-Lab. (France); P. Bertolino, GIPSA-Lab. (France); M. Nicolas, STMicroelectronics (France)

7870 ON Images reconstruction using modified exemplar based method [7870-24]

V. V. Voronin, V. I. Marchuk, South-Russian State Univ. of Economics and Service (Russian Federation); K. O. Egiazarian, Tampere Univ. of Technology (Finland) 
787000 A graph, non-tree representation of the topology of a gray scale image [7870-25]

P. Saveliev, Marshall Univ. (United States)

7870 OP Colour processing in Runge space [7870-26]

A. Restrepo, Univ. de Los Andes (Colombia)

$78700 Q$ Robust image registration for multiple exposure high dynamic range image synthesis [7870-27]

S. Yao, A*STAR Institute for Infocomm Research (Singapore)

INTERACTIVE PAPER SESSION

7870 OR Efficiency analysis of DCT-based filters for color image database [7870-28]

D. V. Fevralev, N. N. Ponomarenko, V. V. Lukin, S. K. Abramov, National Aerospace Univ. (Ukraine); K. O. Egiazarian, J. T. Astola, Tampere Univ. of Technology (Finland)

7870 OS Color image lossy compression based on blind evaluation and prediction of noise characteristics [7870-29]

N. N. Ponomarenko, V. V. Lukin, National Aerospace Univ. (Ukraine); K. O. Egiazarian, Tampere Univ. of Technology (Finland); L. Lepisto, Nokia Corp. (Finland)

7870 OT Unsupervised automated panorama creation for realistic surveillance scenes through weighted mutual information registration [7870-30]

T. P. Keane, E. Saber, H. Rhody, A. Savakis, Rochester Institute of Technology (United States); J. Raj, Lenel Systems International Inc. (United States)

7870 OU Ellipse detection using an improved randomized Hough transformation [7870-31]

Z. Teng, J.-H. Kim, D.-J. Kang, Pusan National Univ. (Korea, Republic of)

7870 OV Detection of motion blur direction based on maxima locations for blind deconvolution [7870-32]

R. M. Chong, T. Tanaka, Tokyo Univ. of Agriculture and Technology (Japan)

7870 OW EM algorithm-based hyperparameters estimator for Bayesian image denoising using BKF prior [7870-33]

L. Boubchir, B. Durning, E. Petit, Lab. Images, Signaux et Systèmes Intelligents, Univ. Paris Est Créteil (France)

7870 OY Color image enhancement algorithm based on logarithmic transform coefficient histogram [7870-35]

J. Xia, K. Panetta, Tufts Univ. (United States); S. Agaian, The Univ. of Texas at San Antonio (United States)

$78700 Z$ Neighbourhood-consensus message passing and its potentials in image processing applications [7870-36]

T. Ružić, A. Pižurica, W. Philips, Ghent Univ. (Belgium)

787010 Alternative method for Hamilton-Jacobi PDEs in image processing [7870-37]

A. Lagoutte, H. Salat, C. Vachier, ENS Cachan, CNRS (France) 
787012 Spatially adaptive alpha-rooting in BM3D sharpening [7870-39]

M. Mäkitalo, A. Foi, Tampere Univ. of Technology (Finland)

787014 Joint distributed source-channel coding for 3D videos [7870-41]

V. Palma, M. Cancellaro, A. Neri, Univ. degli Studi di Roma Tre (Italy)

787015 Simulating images captured by superposition lens cameras [7870-42]

A. S. Thangarajan, R. Kakarala, Nanyang Technological Univ. (Singapore)

787016 Features extraction based on Fisher's information [7870-43]

L. Costantini, P. Sità, Fondazione, Ugo Bordoni (Italy) and Univ. degli Studi di Roma Tre (Italy);

M. Carli, A. Neri, Univ. degli Studi di Roma Tre (Italy)

787017 An improved RANSAC algorithm using within-class scatter matrix for fast image stitching [7870-45]

L. Zhang, Z. Liu, J. Jiao, Graduate Univ. of the Chinese Academy of Sciences (China)

787019 Enhanced bleed through removal for scanned document images [7870-47]

A. Sharma, Hewlett-Packard Research Labs. (India); S. Mahaldar, Shell Inc. (India);

S. Banerjee, Hewlett-Packard Research Labs. (India)

$78701 \mathrm{~A}$ Wavelet-based asphalt concrete texture grading and classification [7870-48]

A. Almuntashri, S. Agaian, The Univ. of Texas at San Antonio (United States)

7870 1B Image segmentation refinement by modeling in turning function space [7870-49]

C. F. S. Volotão, Instituto Militar de Engenharia (Brazil) and Instituto Nacional de Pesquisas Espaciais (Brazil); G. J. Erthal, R. D. C. Santos, L. V. Dutra, Instituto Nacional de Pesquisas Espaciais (Brazil)

7870 1C Integrating ensemble empirical mode decomposition and nonlinear anisotropic diffusion filter for speckle noise reduction in underwater sonar images [7870-50]

S. Bakhtiari, S. Agaian, M. Jamshidi, The Univ. of Texas at San Antonio (United States)

7870 ID Extending JPEG-LS for low-complexity scalable video coding [7870-51]

A. Ukhanova, Technical Univ. of Denmark (Denmark); A. Sergeev, St. Petersburg State Univ. of Aerospace Instrumentation (Russian Federation); S. Forchhammer, Technical Univ. of Denmark (Denmark)

Author Index 


\title{
Conference Committee
}

\author{
Symposium Chair \\ Sabine E. Süsstrunk, École Polytechnique Fédérale de Lausanne \\ (Switzerland) \\ Symposium Cochair
}

Majid Rabbani, Eastman Kodak Company (United States)

Conference Chairs

Jaakko T. Astola, Tampere University of Technology (Finland)

Karen O. Egiazarian, Tampere University of Technology (Finland)

Program Committee

Til Aach, RWTH Aachen (Germany)

Sos S. Agaian, The University of Texas at San Antonio (United States)

Junior Barrera, Universidade de São Paulo (Brazil)

Reiner Creutzburg, Fachhochschule Brandenburg (Germany)

Paul Gader, University of Florida (United States)

Atanas P. Gotchev, Tampere University of Technology (Finland)

John C. Handley, Xerox Corporation (United States)

Vladimir V. Lukin, National Aerospace University (Ukraine)

Stephen Marshall, University of Strathclyde (United Kingdom)

Alessandro Neri, Università degli Studi di Roma Tre (Italy)

Françoise J. Prêteux, TELECOM \& Management SudParis (France)

Giovanni Ramponi, Università degli Studi di Trieste (Italy)

Jagath K. Samarabandu, The University of Western Ontario (Canada)

Akira Taguchi, Musashi Institute of Technology (Japan)

\section{Session Chairs}

$1 \quad$ Image Filtering and Enhancement

Karen O. Egiazarian, Tampere University of Technology (Finland)

2 Image Analysis

Marco Carli, Università degli Studi di Roma Tre (Italy)

3 Image Segmentation and Classification

Sos S. Agaian, The University of Texas at San Antonio (United States) 
$4 \quad$ Image Transforms and Applications

Alessandro Neri, Università degli Studi di Roma Tre (Italy)

$5 \quad$ Image Processing Systems

Karen O. Egiazarian, Tampere University of Technology (Finland)

6 Image Interpolation and Reconstruction

Karen O. Egiazarian, Tampere University of Technology (Finland)

7 Image Representation

Marco Carli, Università degli Studi di Roma Tre (Italy) 